\title{
Relationship between eosinophil cationic pro
and infection intensity in a schistosomiasis endemic community in Ghana
}

This article was published in the following Dove Press journal:

Research and Reports in Tropical Medicine

21 January 2015

Number of times this article has been viewed

Elias Asuming-Brempong ${ }^{1-3}$

Ben Gyan ${ }^{2}$

Abena Serwaa Amoah ${ }^{3,4}$

William van der Puije ${ }^{3,4}$

Langbong Bimi'

Daniel Boakye ${ }^{3}$

Irene $\mathrm{Ayi}^{3}$

'Department of Animal Biology and Conservation Science, University of Ghana, Legon, Ghana; ${ }^{2} I m m u n o l o g y$ Department, ${ }^{3}$ Parasitology

Department, Noguchi Memorial Institute for Medical Research, College of Health Sciences, University of Ghana, Legon, Ghana; ${ }^{4}$ Parasitology Department, Leiden University Medical Centre, Leiden, The Netherlands

\section{Video abstract}

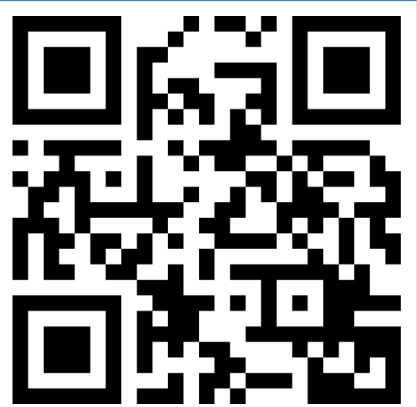

Point your SmartPhone at the code above. If you have a QR code reader the video abstract will appear. Or use: http://dvpr.es//rxaynD

Correspondence: Ben Gyan Noguchi Memorial Institute for Medical Research, University of Ghana, PO Box LG 58I, Legon, Ghana

Tel +233244726016

Fax +233302502182

Email bgyan@noguchi.mimcom.org
Background: Recent studies have shown the urine filtration and Kato-Katz techniques to significantly underestimate infection intensity in Schistosoma haematobium and Schistosoma mansoni infections, respectively. Infection intensity determination by these methods improves only with increasing number of samples collected per participant. This implies tedious and lengthy periods of sample processing and analysis by microscopy examination, hence the increased chances of experimental errors. This study sought to determine the relationship between levels of eosinophil cationic protein (ECP) and infection intensity by egg count both in S. haematobium and S. mansoni single and coinfections.

Methods: This was a cross-sectional study carried out in Pakro, a periurban community in Ghana, involving a total of 308 participants. Each provided urine and stool samples, which were processed using the filtration and Kato-Katz techniques, respectively. Processed samples were examined by microscopy. Aliquots of urine from 73 participants were analyzed for levels of ECP using an ECP enzyme-linked immunosorbent assay kit.

Results: Of the 308 urine samples examined, 59 (19.15\%) were positive for S. haematobium. Significant association was observed between sex and $S$. haematobium infection intensity by egg count $(P<0.05)$ as well as between age and infection intensity $(P<0.001)$. Mean ECP levels were higher in $S$. haematobium-positive samples than in $S$. haematobium-negative samples $(P<0.001)$. There was also positive correlation between ECP and infection intensity (Spearman's $r=0.73, P<0.001$ ).

Conclusion: This study showed that ECP levels are positively associated with infection intensity by egg count in Schistosomiasis infections, even after multivariate adjustment.

Keywords: infection intensity, risk factors, urine filtration, Kato-Katz

\section{Introduction}

An estimated 2 billion people worldwide are affected by schistosomiasis and helminth-related diseases. ${ }^{1}$ More specifically, the trematode Schistosoma $s p$. infects about 200 million people, with an estimated $90 \%$ of the cases occurring in peri-urban and rural areas in Sub-Saharan Africa. ${ }^{2}$ Among the five species known to infect man, Schistosoma mansoni and Schistosoma haematobium are the most prevalent in the continent. Ghana is endemic for schistosomiasis, which is focally distributed in many areas in the country, with some communities reporting a prevalence of $60 \%{ }^{3}$

Stool and urine examination for egg detection by microscopy is currently the WHO-accepted "gold standard" for infection prevalence and intensity determination. However, the need for more accurate indicators of infection intensity determination in Schistosoma spp.-infected individuals has, in recent times, become necessary due to 
the extensive research work done exposing the inadequacies of current methods.

For instance, separate studies in Zanzibar and the People's Republic of China reported low sensitivity of the Kato-Katz technique, which only improved with increasing number of samples., ${ }^{4,5}$ Other studies have reported the collection of between two and four urine samples on consecutive days in efforts to improve the accuracy of measurement of S. haematobium infection prevalence ${ }^{6-8}$ and infection intensity by egg count. ${ }^{9}$

Therefore, complementing current microscopic methods with immunological techniques could significantly improve accurate one-time infection detection and intensity determination of the parasite in single samples.

The measurement of immune molecules or factors in blood, urine, stool, or other secretory/excretory fluid as a way of assessing disease prevalence and infection intensity in schistosomiasis is being extensively explored. ${ }^{8}$ The eosinophil cationic protein (ECP) is one such immunomolecule released during innate immune responses; it is a highly basic and potent cytotoxic single-chain, zinc-containing protein of strong proinflammatory activity, ${ }^{10}$ detectible in blood, stool, and urine, ${ }^{11,12}$ and with a half-life of 45 minutes in circulation. ${ }^{13}$

Penetration of the human skin by the cercaria is done with the aid of secreted proteases and immunomodulators. ${ }^{14}$ Immune responses marking this stage comprise a series of cellular and molecular interactions involving immunoglobulin E-activated mast cells, which release a number of chemical mediators. ${ }^{15}$ These mediators in turn attract increased numbers of eosinophils and other leukocytes to the site of interest, which upon arrival, are induced to release inflammatory mediators and molecules such as ECP, aimed at killing or expelling the invading helminth out of the body. ${ }^{15}$ Detection and measurement of this protein is by means of a polyclonal sandwich-type enzyme-linked immunosorbent assay (ELISA) with an amplification step involving either biotin-avidin-peroxidase ${ }^{16}$ or horseradish peroxidase. ${ }^{17}$

ECP is released locally, usually in the presence of either a helminth or an allergen. Hence, increased worm burden could lead to increased eosinophilia, and eventually increased levels of ECP in circulation at measurable levels. Measured levels can be compared with the egg counts in urine and stool, which is the current WHO gold standard. Although the search for a highly-specific, sensitive, cost-effective, noninvasive diagnostic assay for schistosomiasis is far from over, studies into immune molecules such as ECP may lead a step further in that direction. The aim of this work, therefore, is to explore the relationship between ECP levels in urine and infection intensity by microscopy.

\section{Materials and methods}

\section{Study site and subjects}

The study was conducted in Pakro, a periurban community along the Densu River in the Eastern Region of Ghana. The population of the community is about 5,000 and is ethnically diverse. A pilot study conducted a few months prior to the commencement of this work showed the prevalence of S. haematobium to be at $40 \%$. This involved the collection, processing, and analysis of 100 urine samples from randomlyselected members within the Pakro community (data not shown), out of which 40 were found to be infected by egg detection via microscopy. Available water sources apart from the Densu River were a borehole and a number of hand-dug wells.

\section{Study design}

A cross-section of participants between the ages of 6 and 96 years were randomly recruited to participate in the study. Informed consent and authorization was obtained from the disease control officer at the Pakro Health Centre, the chief and opinion leaders of the community, as well as adult participants and parents of wards involved in the study. Study participants provided one stool and one urine sample each, which were screened for S. mansoni and S. haematobium infections. Participants also, via questionnaire interviews, provided information on treatment history for bilharzia and other soil-transmitted helminthes, their frequency of contact with the Densu River, and history for asthma and allergies. Data was analyzed using Microsoft Office Excel 2007, Graph Pad Prism version 5.04, and SPSS version 16.0 software.

\section{Sample collection and analysis}

Up to $50 \mathrm{~mL}$ of urine and a minimum of $2 \mathrm{~g}$ of stool sample were provided by each participant in clean, well-labeled falcon tubes and containers provided by the study. Urine samples were collected between 10.00 am and $2.00 \mathrm{pm}$ while stool samples were collected in the mornings. Stool and urine samples from these participants were processed using the Kato-Katz and filtration methods, respectively.

\section{Stool sample preparation}

The Kato-Katz technique was conducted using a stool examination kit (Vestergaard Frandsen, Denmark). Briefly, a small amount of well-mixed fecal matter from the sample was placed on a piece of pergamon paper with the aid of a spatula. A nylon screen was placed on top of the fecal matter and scraping was done with the spatula to collect sieved feces. A plastic template, positioned centrally on a microscope glass slide, was filled in the middle aperture with a small amount 
of the sieved feces. The template was carefully removed, and the fecal matter on the slide was covered with a cellophane strip soaked in a malachite green/glycerol mixture. This preparation was inverted and pressed down against a hard, flat surface for even spread of fecal matter and was left to clear under direct sunlight for 30 minutes, followed by microscopic examination for hookworm eggs. ${ }^{18}$

\section{Formol-ether concentration method}

The formol-ether technique was performed as a confirmatory test on stool samples negative for $S$. mansoni. This involved the thorough emulsifying of the remaining feces sample with $7 \mathrm{ml}$ of $10 \%$ formol-saline, and then straining through cotton gauze into a centrifuge tube, using a funnel. A maximum of
$3 \mathrm{~mL}$ of ether was added and the mixture was shaken vigorously for 1 minute. The mixture was then centrifuged at a speed of $2,000 \mathrm{rpm}$ for 5 minutes, and the debris at the interface of the two liquids was loosened from the wall of the tube with a stick. All the liquid supernatant was decanted, and the upper part of the tube wiped clear of fatty debris. The small deposit at the bottom of the tube was dislodged and resuspended, transferred onto a slide with the aid of a Pasteur pipette, and covered with a glass slip for microscopic examination. ${ }^{18}$

\section{Urine sample preparation}

Urine filtration was conducted using a filtration apparatus and membrane of pore size $10 \mu \mathrm{m}$ (Millipore, Ireland) as well as a $10 \mathrm{~mL}$ syringe. About $10 \mathrm{ml}$ of urine sample was aspirated

Table I General characteristics of study population

\begin{tabular}{|c|c|c|c|c|}
\hline & $\begin{array}{l}\text { Schistosoma haematobium- } \\
\text { Infected, n (\%) }\end{array}$ & $\begin{array}{l}\text { Uninfected, } \\
\text { n (\%) }\end{array}$ & $\begin{array}{l}\text { Subset } \\
\text { Total (N) }\end{array}$ & $\begin{array}{l}\text { Subset Total }(\mathbf{N}) / \\
\text { Grand Total }\left(\mathbf{N}_{\mathrm{T}}\right) \\
{\left[\mathrm{N} / \mathbf{N}_{\mathrm{T}}(\%)\right]}\end{array}$ \\
\hline \multicolumn{5}{|l|}{ General information } \\
\hline \multicolumn{5}{|l|}{ Demographics } \\
\hline \multicolumn{5}{|l|}{ Age (years) } \\
\hline $6-10$ & II (I6.40) & $56(83.58)$ & 67 & $67 / 308(21.80)$ \\
\hline $11-15$ & $22(24.70)$ & $67(75.30)$ & 89 & $89 / 308$ (28.89) \\
\hline $16-20$ & $21(35.60)$ & $38(64.40)$ & 59 & $59 / 308(19.15)$ \\
\hline $21-25$ & $2(12.50)$ & $14(87.50)$ & 16 & $16 / 308(5.19)$ \\
\hline$>25$ & $3(3.90)$ & $74(96.10)$ & 77 & $77 / 308(25.00)$ \\
\hline \multicolumn{5}{|l|}{ Sex } \\
\hline Male & $4 \mathrm{I}(25.95)$ & $117(74.10)$ & 158 & I58/308 (51.30) \\
\hline Female & $18(12.00)$ & I $32(88.00)$ & 150 & I50/308 (48.70) \\
\hline \multicolumn{5}{|c|}{ Frequency of Contact (Questionnaire) } \\
\hline Once a month & $10(4 I .67)$ & $14(58.33)$ & 24 & 24/308 (7.79) \\
\hline Once a week & $13(31.70)$ & $28(68.29)$ & 41 & $4 \mid / 308(|3.3|)$ \\
\hline Everyday & $6(20.69)$ & $23(79.31)$ & 29 & 29/308 (9.42) \\
\hline \multicolumn{5}{|c|}{ Infection and treatment history (questionnaire) } \\
\hline \multicolumn{5}{|c|}{ Food-related allergies } \\
\hline Yes & $8(19.50)$ & $33(80.50)$ & 41 & $4 I / 308(|3.3|)$ \\
\hline No & $5 I(19.10)$ & $216(80.90)$ & 267 & $267 / 308(86.70)$ \\
\hline \multicolumn{5}{|l|}{ Asthma } \\
\hline Yes & $3(17.60)$ & $14(82.40)$ & 17 & $17 / 308(5.52)$ \\
\hline No & $56(19.20)$ & $235(80.76)$ & 291 & $291 / 308(94.48)$ \\
\hline \multicolumn{5}{|l|}{ Treatment histories } \\
\hline Schistosomiasis & $2(13.30)$ & $13(86.70)$ & 15 & I5/308 (4.87) \\
\hline Soil-transmitted helminths & $17(1 \mathrm{l} .40)$ & $132(88.60)$ & 149 & |49/308 (48.38) \\
\hline Asthma & $\mathrm{I}(\mathrm{I} 2.50)$ & $7(87.50)$ & 8 & $8 / 308(2.60)$ \\
\hline Allergies & $0(0.00)$ & $2(100.00)$ & 2 & $2 / 308(0.65)$ \\
\hline \multicolumn{5}{|l|}{ Other parasitology results } \\
\hline \multicolumn{5}{|c|}{ Soil-transmitted helminths (STHs) } \\
\hline Ascaris lumbricoides & $0(0)$ & I (I00.00) & 1 & $1 / 308(0.32)$ \\
\hline Trichuris trichiura & $0(0)$ & I (I00.00) & 1 & I/308 (0.32) \\
\hline Necator americanus & I (I2.50) & $7(87.50)$ & 8 & $8 / 308(2.60)$ \\
\hline $\begin{array}{l}\text { Schistosoma mansoni } \\
\text { (eggs/10 mL urine) }\end{array}$ & $2(66.70)$ & I (33.30) & 3 & I/308 (0.32) \\
\hline \multicolumn{5}{|l|}{ Microhematuria } \\
\hline Present & $36(56.30)$ & $28(43.80)$ & 64 & 64/308 (20.78) \\
\hline Absent & $23(9.230)$ & $221(88.80)$ & 249 & 249/308 (80.84) \\
\hline
\end{tabular}


Table 2 Intensity of Schistosoma haematobium and Schistosoma mansoni infections among study participants

\begin{tabular}{|c|c|c|c|}
\hline $\begin{array}{l}\text { Age group } \\
\text { (years) }\end{array}$ & $\begin{array}{l}\text { Light infection, } \\
\text { n (\%) }\end{array}$ & $\begin{array}{l}\text { Heavy infection, } \\
\text { n (\%) }\end{array}$ & $\begin{array}{l}\text { Total } \\
(\mathbf{N}) \\
\end{array}$ \\
\hline \multicolumn{4}{|c|}{ S. haematobium } \\
\hline $6-10$ & $10(90.9)$ & I (9.0) & 11 \\
\hline $11-15$ & I8 (8I.8) & $4(18.2)$ & 22 \\
\hline $16-20$ & 17 (80.9) & $4(19.0)$ & 21 \\
\hline $21-25$ & $2(100.0)$ & $0(0)$ & 2 \\
\hline$>25$ & $3(100.0)$ & $0(0)$ & 3 \\
\hline \multicolumn{4}{|l|}{ S. mansoni } \\
\hline$>25$ & I (I00.0) & $0(0)$ & 1 \\
\hline \multicolumn{4}{|c|}{ S. haematobium/S. mansoni } \\
\hline $6-10$ & I (I00.0) & $0(0)$ & 1 \\
\hline $16-20$ & I (100.0) & $0(0)$ & I \\
\hline
\end{tabular}

with the syringe after shaking the sample in the tube to mix. This was forced through an aperture in the filtration apparatus containing the membrane. The membrane was carefully removed using a pair of forceps and placed on a clean, welllabeled glass slide with the dull surface up. The preparation was left to dry, and a drop of saline solution was applied to the membrane prior to microscopic examination. ${ }^{19}$

\section{Sedimentation technique}

This was also performed as a confirmatory test on urine samples negative for $S$. haematobium. Hence, the remaining urine sample was centrifuged at 2,000 rpm for 10 minutes and quickly decanted. The sediments were then examined by microscopy to confirm negativity of egg presence, or otherwise. Sediments to be examined later were stored at $4^{\circ} \mathrm{C} .{ }^{20}$

\section{ECP ELISA}

Measurement of ECP in urine was undertaken using an ECP ELISA kit (MBL International, USA) according to manufacturer's intructions. ${ }^{21}$ Briefly, urine samples were mixed with an assay diluent provided in the kit and then transferred to a 96-well microplate precoated with anti-human ECP antibody. After incubation and washing, $100 \mu \mathrm{L}$ of horseradish peroxidase-conjugated anti-human
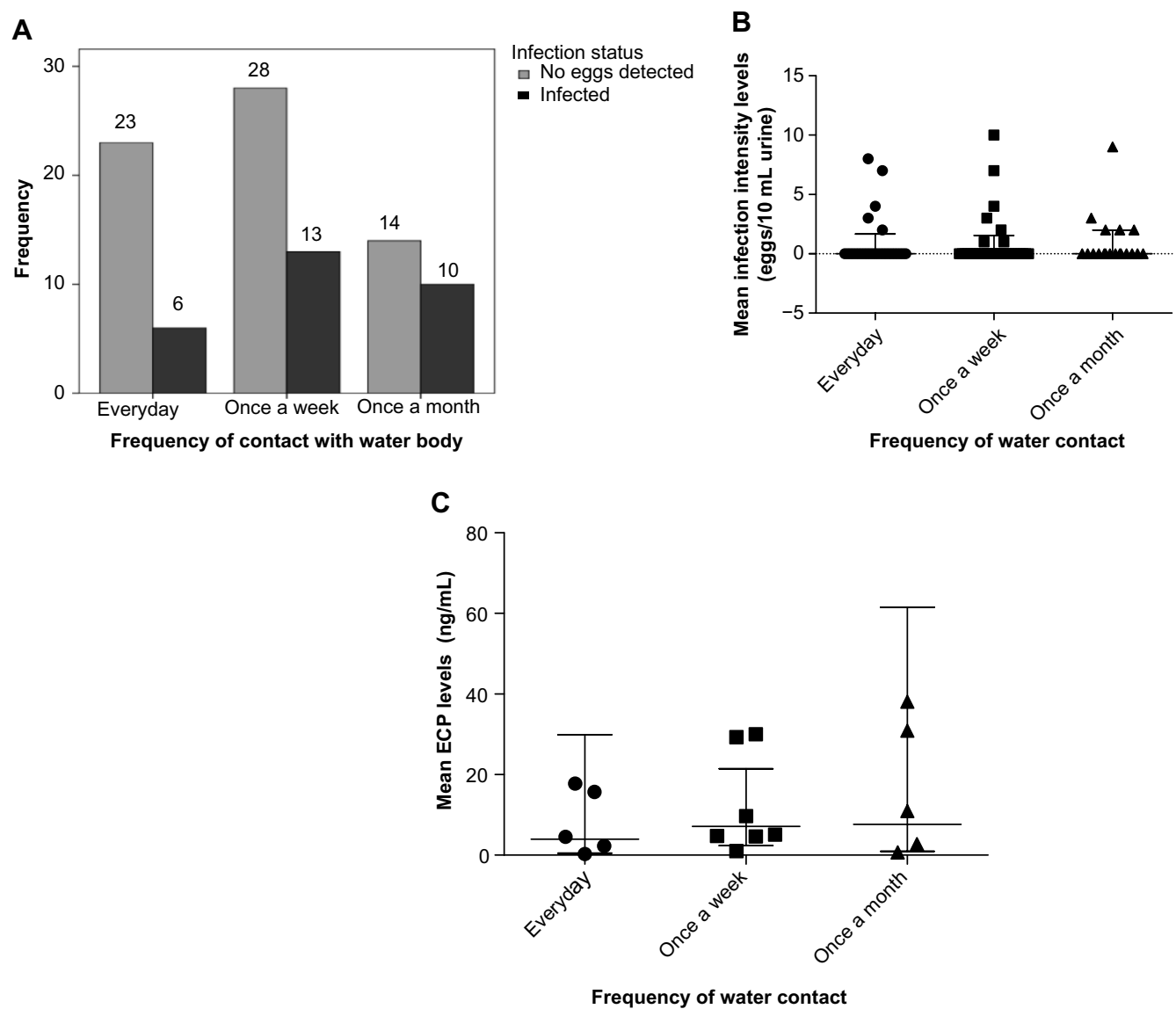

Figure I Schistosoma haematobium prevalence distribution, mean infection intensity, and mean ECP level distribution.

Notes: $S$. haematobium prevalence distribution by frequency of contact with water body among study participants $(\mathbf{A})$. Distribution was not found to be significant $(P=0.255)$. Mean infection intensity $(\mathbf{B})$ and mean ECP level distribution $(\mathbf{C})$ by frequency of contact with water body among study participants. Distribution in both cases were found to be insignificant $(P=0.238$ and $P=0.743$, respectively).

Abbreviation: ECP, eosinophil cationic protein. 
ECP polyclonal antibody was added and followed with the substrate reagent tetramethylbenzidine $/ \mathrm{H}_{2} \mathrm{O}_{2}$. All standards used in the assay were provided in the kit. Absorbance was read at $450 \mathrm{~nm}$ using an ELISA microplate reader (BioTek) and the results interpreted in accordance with the protocol provided. ${ }^{17}$

Due to the limited resources available for the assay, a stringent criterion was prepared and adhered to. First, it was ensured that participants whose samples were selected for this part of the study had complete parasitological data (ie, both urine and stool samples had been provided). Second, none of the participants, according to questionnaire response, had had a history of allergies or asthma. Third, it was ensured that no soil-transmitted helminths were found in their corresponding stool samples. In all, 73 samples out of the 308 collected were selected for the ECP ELISA.

\section{Analysis of data}

Data input was done using Microsoft Office Excel 2007 and exported to SPSS version 16.0 for analysis. Some of the graphs were drawn using Graph Pad Prism version 5.04. In order to determine whether ECP levels correlated strongly and positively with infection intensity, a description on the general characteristics of the data including age, infection status, sex, and ECP levels was first made. Next, potential confounders to the main relationship outlined above were identified and tested using logistic regression analysis for nonparametric data. Individual associations between nonscale variables were determined using the Mann-Whitney $U$ rank sum and the Kruskal-Wallis tests. The Spearman's rank correlation was used in determining the correlation between infection intensity by egg count and ECP levels.

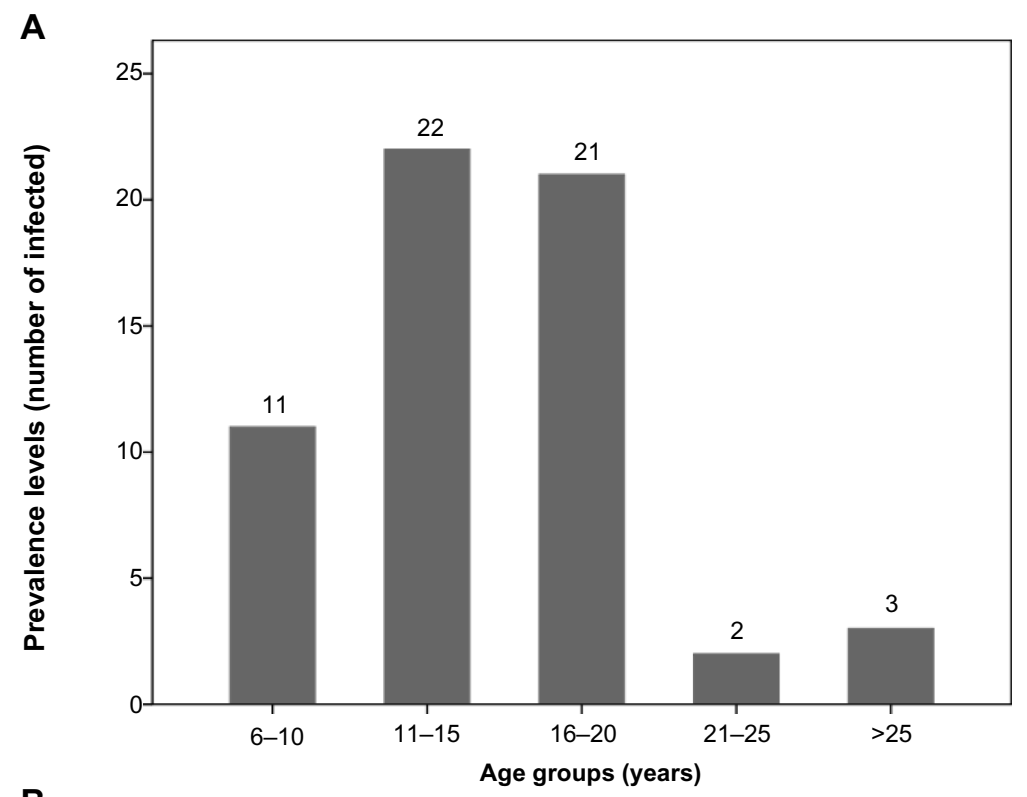

B

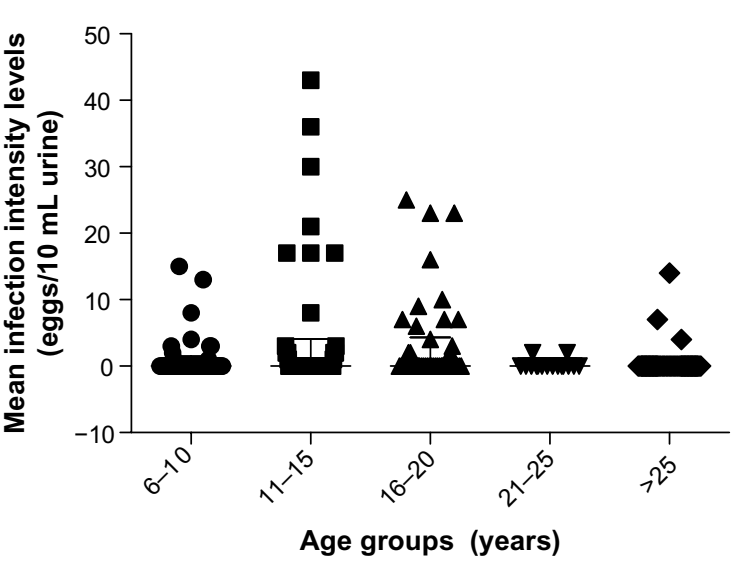

Figure 2 Age distribution of prevalence of infection $(\mathbf{A})$ and mean intensity of infection $(\mathbf{B})$ among study participants. Note: There were significant differences in both prevalence $(P<0.00 \mathrm{I})$ and mean intensity $(P<0.00 \mathrm{I})$. 


\section{Results}

\section{Characteristics of study population}

A total of 308 participants took part in the study, out of whom $158(51.30 \%)$ were males, and 150 (48.70\%) were females. The ages of these participants ranged between 6 and 96 years, with 233 (75.65\%) of them falling between the ages of 6 and 26 years. The mean age among the study participants was 23.12 years.

From the questionnaire data, 127 (41.23\%) of the participants reported having contact with the Densu River, with $41(13.31 \%)$ of them doing so on a weekly basis. Prevalence rates were, however, found to have no statistically significant relationship with frequency of contact with the water body ( $P=0.26)$. About half $(149,48.38 \%)$ of the participants affirmed treatment against intestinal worms. Only 15 (4.87\%) participants reported having been treated for schistosomiasis. Questionnaire results also indicated 17 (5.52\%) participants had asthma, eight of whom claimed to be receiving treatment. Also, 41 (13.31\%) participants stated they had food-related allergies, two of whom reported to be receiving treatment at the time of sampling (Table 1).

\section{Parasitological data}

Microscopic examinations of prepared urine samples showed a total of $59(23.23 \%)$ S. haematobium infections distributed among the various age groups (Table 2). Of this number, 50 (19.69\%) were light and nine (3.54\%) were heavy infections. Mean infection intensity was found to be 4.97 eggs $/ 10 \mathrm{~mL}$ urine (standard deviation [SD] =25.71). Additionally, the highest mean infection intensity levels were observed for participants who, according to questionnaire data, had daily contact with the Densu River (Figure 1B), though this was not statistically significant $(P=0.24)$. Other helminths, Ascaris lumbricoides (hookworm) and Trichuris trichiura were identified in ten of the stool samples examined by microscopy.

\section{Hematuria}

Urinalysis reagent strips (Shenzhen Mindray Bio-Medical Electronics Co, Ltd) were used for the detection of hematuria in the urine samples of participants. Out of the 308 urine samples analyzed, 64 (20.78\%) were positive for hematuria. Of this number, $36(56.3 \%)$ were found positive for $S$. haematobium eggs (Table 1).

\section{S. haematobium prevalence and infection intensity in age groups}

The prevalence distribution pattern of $S$. haematobium infection among the various age groups as shown in Figure 2A

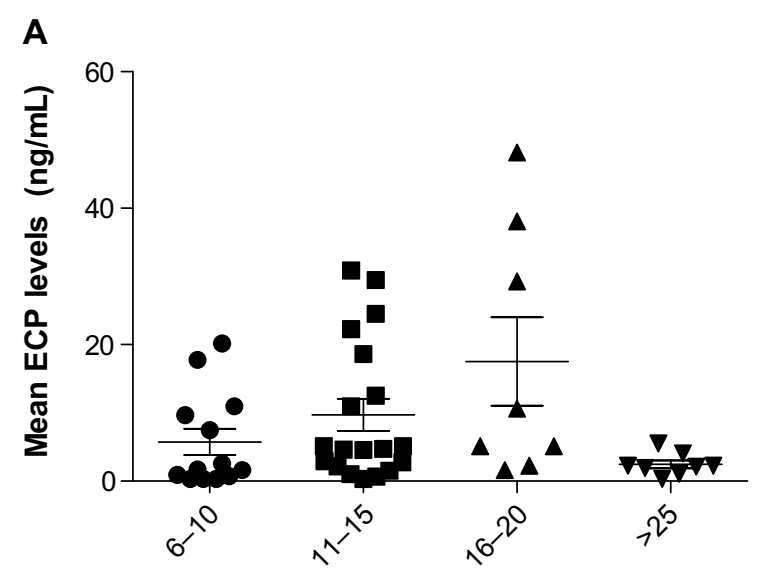

Age groups (years)

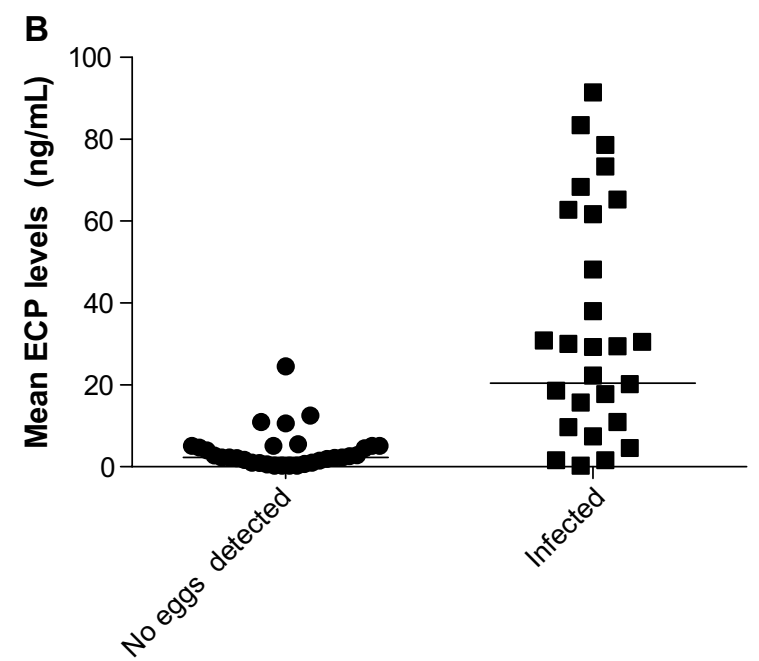

Infection status

Figure 3 Mean ECP level distribution by age groups $(\mathbf{A})$ and infection status (B) among study participants.

Note: Differences were significant in infection status $(P<0.001)$ but not in age groups $(P=0.067)$.

Abbreviation: $\mathrm{ECP}$, eosinophil cationic protein

was found to be almost identical to that for mean infection intensity (Figure 2B). In both cases, there was a rise in levels, peaking in the 11-15-year age group, and then declining significantly for the subsequent age groups. The lowest prevalence and mean intensity levels were observed among participants in the 21-25-year age group.

\section{ECP ELISA results}

Of the 73 samples selected for the ECP ELISA, 44 were positive for $S$. haematobium infections (by filtration), 29 were Schistosoma spp. negative (indicated by filtration and confirmed by sedimentation), two were positive for both $S$. haematobium and S. mansoni, and one was positive for $S$. mansoni. The mean ECP levels among the $S$. haematobium-infected were found to be significantly higher $(P<0.001)$ than in uninfected 
$r=0.7236 ; P<0.0001$

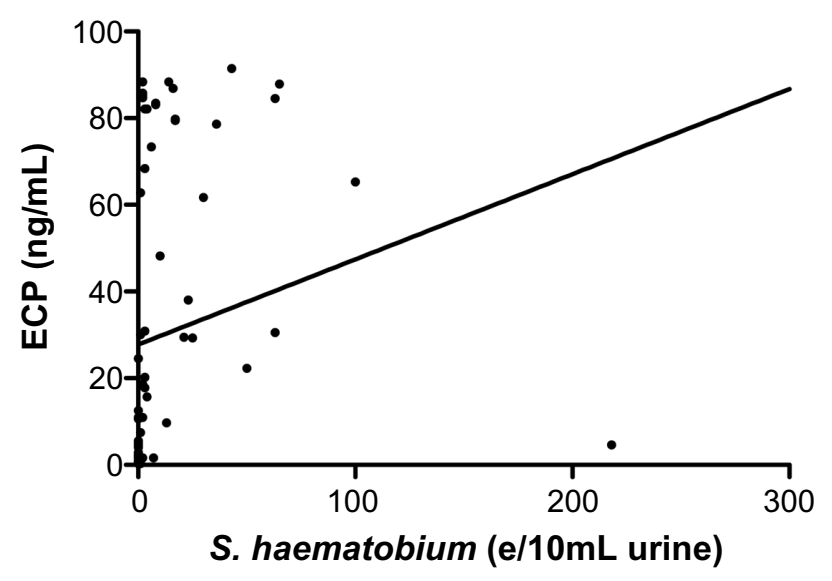

Figure 4 Correlation between ECP $(\mathrm{ng} / \mathrm{ml})$ and infection intensity $(\mathrm{eggs} / \mathrm{l} 0 \mathrm{ml}$ urine).

Note: Differences were positive (Spearman's $r=0.724 ; P<0.001$ ).

Abbreviations: ECP, eosinophil cationic protein; Sh, Schistosoma haematobium.

participants (Figure 3B). The association between mean ECP levels and age were found to be statistically insignificant (Figure 3A). The same was true when the relationship between mean ECP levels and frequency of contact with the water body was measured $(P=0.73)$.

\section{Relationship between other risk factors and infection intensity}

Firstly, the Spearman's the correlation between infection intensity (eggs/10 mL urine) and ECP levels (Figure 4) was found to be positive ( $r=0.73, P<0.001)$. Furthermore, the relationships between infection intensity by egg count and the study variables, namely sex and age, were determined so as to rule them out as potential confounders.

The Mann-Whitney $U$ test was used to determine the strength of association between sex and $S$. haematobium infection intensity by egg count. Sex was found to have a significant association with infection intensity $(P<0.05)$, which was higher among male than female participants (Figure 5A). Mean ECP levels were also found to be higher among the male participants, though this was not statistically significant (Figure 5B). Via the Kruskal-Wallis and Mann-Whitney $U$ tests, respectively, age was found to associate significantly with infection intensity by egg count $(P<0.001)$ but not with ECP $(P=0.067)$. The Multivariate logistic regression model was used to test the combined effects of age and sex on the $\mathrm{ECP} /$ infection intensity relationship, which was found to still be significant after multivariate adjustment $(P<0.05)$.

\section{Discussion}

This study aimed at investigating the relationship between ECP levels and infection intensity by egg count. S. haematobium
A

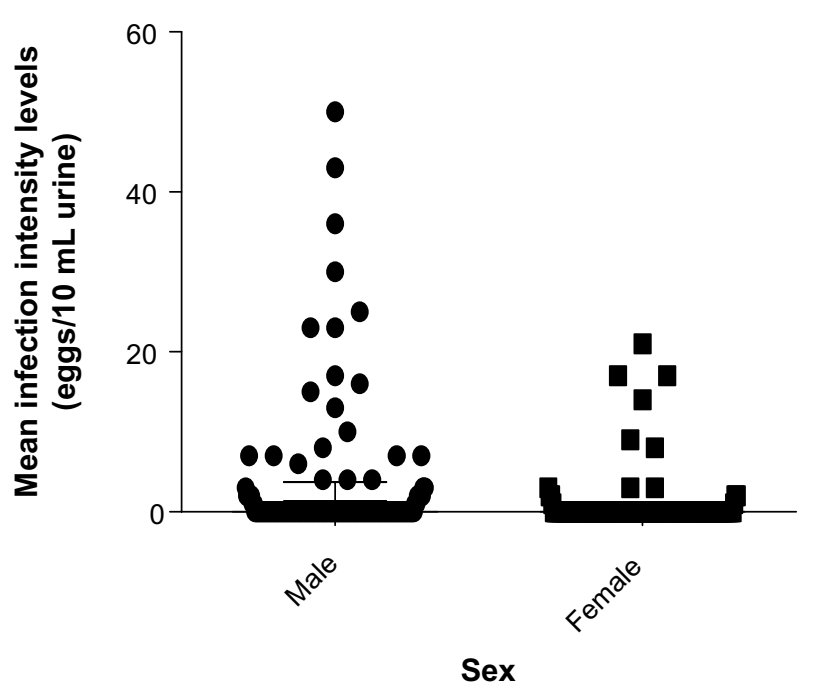

B

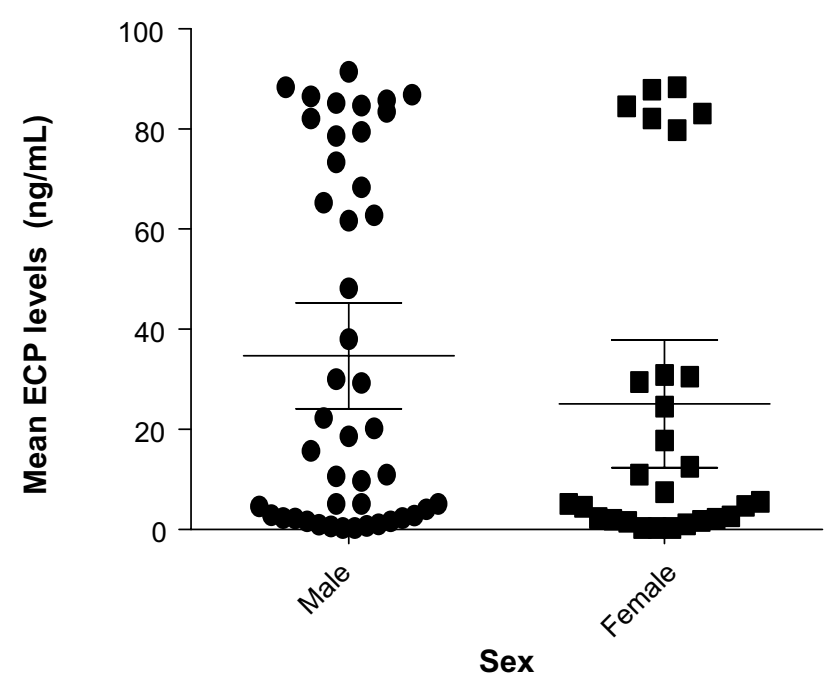

Figure 5 Mean infection intensity (A) and mean ECP level (B) distribution by sex among study participants.

Notes: Differences were significant in mean infection intensity by egg count between male and female participants $(P<0.05[\mathbf{A}])$; but not with ECP levels $(P=0.33[\mathbf{B}])$.

Abbreviation: ECP, eosinophil cationic protein.

was found to be the dominant schistosomal infection among the inhabitants of Pakro. Of the 308 urine samples collected, only one was detected with $S$. mansoni eggs, and two with both $S$. haematobium and $S$. mansoni eggs. These S. mansoniinfected samples may therefore be foreign or residuals of a previous infection. Stool sample analysis yielded no positives, including those which had $S$. mansoni egg detections in corresponding urine samples.

From our study, it is clear that Pakro is a low-prevalence community. According to the WHO, prevalence and infection intensity of schistosomiasis in an area is deemed low when prevalence is below $50 \%$ and the proportion of heavilyinfected $(>50$ eggs $/ 10 \mathrm{~mL}$ urine $)$ participants is below $10 \%{ }^{22}$ 
Our study revealed Pakro to have an infection prevalence of $40 \%$ (among the 308 participants sampled), with $2.9 \%$ of the participants heavily infected. Mean infection intensity and prevalence were found to be weakly associated with frequency of contact with the Densu River $(P=0.24$ and $P=0.26$, respectively). Prevalence for other soil-transmitted helminths, including Necator americanus, A. lumbricoides, and $T$. trichiura, was found to be low as well, with $10(3.2 \%)$ out of the 308 participants testing positive for at least one of these helminths. Additionally, among participants found positive for $S$. haematobium, 56.3\% were found positive for hematuria. Interestingly, among participants found positive for hematuria, for instance, $43.8 \%$ of them were $S$. haematobium negative by microscopy. Cavalcanti et $\mathrm{al}^{23}$ reported that the sensitivity of parasitological detection techniques decreased considerably in low-endemic communities, where egg excretion among participants was low. Hence, it is possible that the $43.8 \%$ of participants negative for infection but positive for hematuria may actually be positive for $S$. haematobium. However, due to the great fluctuations of egg output, ${ }^{24}$ they may not have shed eggs at the time of providing the sample.

Our results showed an initial increase in infection intensity with age which peaked in the 11-15-year age group and then declined with further increase in age (Figure 2B). Aryeetey et al, ${ }^{25}$ in studies conducted in a community along the same Densu River, reported intensity of infection being highest among children aged 10-14 years. This trend is also similar to that observed by Mutapi et al, ${ }^{26}$ who reported a peak in infection intensity between the ages of 10 and 12 years. Prevalence of $S$. haematobium infection was also found to be highest among participants in the 11-15-year age group, implying that a higher number of participants within this age group are infected with $S$. haematobium than in any other age group (Figure 2A). All in all, our study supports observations ${ }^{27,28}$ which indicate that children are more easily predisposed to schistosomiasis infection than adults.

Mean ECP levels were significantly higher in S. haematobium-positive cases than in S. haematobiumnegative cases. This is similar to results reported elsewhere. ${ }^{8}$ Frequency of contact was, however, found to be insignificantly associated with mean ECP levels $(P=0.74)$ (Figure 1C). Additionally, association between ECP and sex was insignificant $(P=0.33$ ) (Figure 5B). Albeit, males were generally found to have higher ECP levels than females.

ECP was found to correlate positively with infection intensity (Figure 5A) in the absence of possible confounding factors. The association remained strong after adjusting for sex and age. The reduced infection intensity could be indicative of the onset of natural immunity developed over the years as a result of repeated exposure, ${ }^{27}$ and secondly due to declining activity resulting from aging. The sharp decline in infection intensity levels among participants 16 years and older (Figure 2B) may also be indicative of a state of chronic schistosomiasis, which is characterized by near-cessation of the voiding of eggs. Interestingly, ECP levels peak in the 16-20-year age group (Figure 3A), suggesting the onset of immune response, with the production of immunoglobulin $\mathrm{E}$ and the eventual increase in ECP levels in circulation. The sharp decline in later year groups could be indicative of the onset of regulatory immune responses, characterized by the significant increase in levels of interleukin-10 and T-regulatory cell populations in blood circulation. ${ }^{21}$ Leutscher et $\mathrm{al}^{29}$ had also observed a positive correlation between egg excretion and ECP levels.

Based on research findings on the association of allergies and asthma to ECP, ${ }^{30,31}$ subjects were interviewed on disease history with respect to asthma and allergies; the relationship of these with ECP as well as with infection intensity was also determined using logistic regression analysis (table not shown). The results generated, however, showed no significant influence of allergies or asthma on either infection intensity by egg count or infection status both in the univariate and multivariate assessments. It must also be noted that analysis was based purely on questionnaire responses, and hence might not indicate the true status of these allergic diseases in the population.

Out of the 254 samples tested, two were found via urine sample analysis to be positive for both $S$. haematobium and S. mansoni eggs. Quality assurance checks were done by experienced technicians to ensure validity of claims. Though these were excluded from analysis, the mean ECP levels obtained from these two samples $(84.86, \mathrm{SD}=2.43$ ) was almost double the levels obtained for $S$. haematobium samples (50.31, $\mathrm{SD}=32.55$ ) only. It would therefore be interesting to ascertain ECP levels in S. haematobium/S. mansoni coinfections against those in single schistosomiasis infections.

\section{Conclusion}

ECP levels were found to correlate positively and significantly with infection intensity. ECP was also found to be positively associated with infection status. The effects of potential confounders, including sex and age were tested and found, after multivariate adjusting, to have little effect on ECP/infection intensity association. Also, mean infection intensity by egg count was found to be higher among male participants. 
Further studies on testing the sensitivity and specificity of ECP in disease and infection intensity diagnosis with the aim of developing a detection strip for quick diagnosis in urine samples should be carried out. This would enhance field work and minimize invasive research methodologies.

\section{Acknowledgments}

We are grateful to the staff of the Parasitology and Immunology Departments of Noguchi Memorial Institute for Medical Research, particularly Ms Akua Attafua, Messrs Jonas Asigbee (of blessed memory), Joseph Okyere, Sampson Otoo, and Bright Adu for facilitating the research. We would also like to thank Prof Maria Yazdanbakhsh, Ms Abena Serwaa Amoah, and the SchistoINIR project team for providing personnel, intellectual, technical, as well as logistic support for the study.

\section{Disclosure}

The authors report no conflicts of interest in this work.

\section{References}

1. World Health Organization. Helminth Control in School-Age Children: A Guide for Managers of Control Programmes. 2nd ed. Geneva: World Health Organization; 2011. Available from: http://whqlibdoc.who.int/ publications/2011/9789241548267_eng.pdf. Accessed October 29, 2013.

2. World Health Organization. First WHO report on neglected tropical diseases: working to overcome the global impact of neglected tropical diseases [webpage on the Internet]. Geneva: World Health Organization; 2010:129-130. Available from: http://www.who.int/ neglected_diseases/2010report/en/. Accessed October 20, 2013.

3. Obeng BB, Aryeetey YA, de Dood CJ, et al. Application of a circulatingcathodic-antigen (CCA) strip test and real-time PCR, in comparison with microscopy, for the detection of Schistosoma haematobium in urine samples from Ghana. Ann Trop Med Parasitol. 2008;102(7):625-633.

4. Knopp S, Stothard JR, Rollinson D, et al. From morbidity control to transmission control: time to change tactics against helminths on Unguja Island, Zanzibar. Acta Trop. 2013;128(2):412-422.

5. Lin DD, Liu JX, Liu YM, et al. Routine Kato-Katz technique underestimates the prevalence of Schistosoma japonicum: a case study in an endemic area of the People's Republic of China. Parasitol Int. 2008;57(3):281-286.

6. Brouwer KC, Ndhlovu PD, Wagatsuma Y, Munatsi A, Shiff CJ. Epidemiological assessment of Schistosoma haematobium-induced kidney and bladder pathology in rural Zimbabwe. Acta Trop. 2003;85(3): 339-347.

7. Kosinski KC, Adjei MN, Bosompem KM, et al. Effective control of Schistosoma haematobium infection in a Ghanaian community following installation of a water recreation area. PLoS Negl Trop Dis. 2012;6(7):e1709.

8. Reimert CM, Mshinda HM, Hatz CF, et al. Quantitative assessment of eosinophiluria in Schistosoma haematobium infections: a new marker of infection and bladder morbidity. Am J Trop Med Hyg. 2000;62(1):19-28.

9. Bourke $\mathrm{CD}$, Nausch $\mathrm{N}$, Rujeni $\mathrm{N}$, et al. Integrated analysis of innate, Th1, Th2, Th17, and regulatory cytokines identifies changes in immune polarisation following treatment of human schistosomiasis. J Infect Dis. 2012;208(1):159-169.

10. Reimert CM, Venge P, Kharazmi A, Bendtzen K. Detection of eosinophil cationic protein (ECP) by an enzyme-linked immunosorbent assay. J Immunol Methods. 1991;138(2):285-290.
11. Niedworok M, Sordyl B, Borecka A, Gawor J, Małecka-Panas E. [Estimation of eosinophilia, immunoglobulin $\mathrm{E}$ and eosinophilic cationic protein concentration during the treatment of toxocariasis]. Wiad Parazytol. 2008;54(3):225-230. Polish.

12. Reimert CM, Tukahebwa EM, Kabatereine NB, Dunne DW, Vennervald BJ. Assessment of Schistosoma mansoni induced intestinal inflammation by means of eosinophil cationic protein, eosinophil protein $\mathrm{X}$ and myeloperoxidase before and after treatment with praziquantel. Acta Trop. 2008;105(3):253-259.

13. Koller DY. Sampling methods: urine/blood analysis. Am J Respir Crit Care Med. 2000;162(2 Pt 2):31-33.

14. Curwen RS, Ashton PD, Sundaralingam S, Wilson RA. Identification of novel proteases and immunomodulators in the secretions of schistosome cercariae that facilitate host entry. Mol Cell Proteomics. 2006;5(5): 835-844.

15. Parham P. The Immune system (2nd Edition); Taylor and Francis; Abingdon, England, UK; 2004;318-319.

16. Reimert CM, Fitzsimmons CM, Joseph S, et al. Eosinophil activity in Schistosoma mansoni infections in vivo and in vitro in relation to plasma cytokine profile pre- and posttreatment with praziquantel. Clin Vaccine Immunol. 2006;13(5):584-593.

17. Medical and Biological Laboratories Co, Ltd. Quantitative Test Kit for Human ECP MESACUP ECP TEST. Code No 7618E. 2006:1-7. Available from: http://www.mblintl.com/products/datasheets/7618E_ MESACUP_ECP_Test_(ENG)_2013.12.9.pdf. Accessed July 20, 2012.

18. World Health Organization, Division of Control of Tropical Diseases, Schistosomiasis and Intestinal Parasites Unit. Training Manual on Diagnosis of Intestinal Parasites: Tutor's Guide. Geneva: World Health Organization; 2004:28-29.

19. Peters PA, Mahmoud AA, Warren KS, Ouma JH, Siongok TK. Field studies of a rapid, accurate means of quantifying Schistosoma haematobium eggs in urine sample. Bull World Health Organ. 1976;54(2): $159-162$.

20. Cheesebrough M. District Laboratory Practice in Tropical Countries, Part 1. New Delhi, India: Cambridge University Press; 2009 : 237-239.

21. Maizels RM, Yazdanbakhsh M. Immune regulation by helminth parasites: cellular and molecular mechanisms. Nat Rev Immunol. 2003;3(9):733-744.

22. World Health Organization. Prevention and control of schistosomiasis and soil-transmitted helminthiasis: report of a WHO expert committee. Technical Report series 912. Geneva: World Health Organization; 2002:31, 33 .

23. Cavalcanti MG, Silva LF, Peralta RH, Barreto MG, Peralta JM. Schistosomiasis in areas of endemicity: a new era in diagnosis. Trends Parasitol. 2013;29(2):75-82.

24. Bosompem KM, Owusu O, Okanla EO, Kojima S. Applicability of a monoclonal antibody-based dipstick in diagnosis of urinary schistosomiasis in the Central Region of Ghana. Trop Med Int Health. 2004;9(9): 991-996.

25. Aryeetey ME, Wagatsuma Y, Yeboah G, et al. Urinary schistosomiasis in southern Ghana: 1. Prevalence and morbidity assessment in three (defined) rural areas drained by the Densu river. Parasitol Int. 2000;49(2):155-163.

26. Mutapi F, Winborn G, Midzi N, Taylor M, Mduluza T, Maizels RM. Cytokine responses to Schistosoma haematobium in a Zimbabwean population: contrasting profiles for IFN-gamma, IL-4, IL-5 and IL-10 with age. BMC Infect Dis. 2007;7:139.

27. Kabatereine NB, Vennervald BJ, Ouma JH, et al. Adult resistance to schistosomiasis mansoni: age-dependence of reinfection remains constant in communities with diverse exposure patterns. Parasitology. 1999;118 (Pt1):101-105.

28. Tetteh IK, Tetteh K, Adjei RO, Sasu S, Appiah-Kwakye L. Index of potential contamination: Schistosoma haematobium infections in school children in the Ashanti Region of Ghana. East Afr Med J. 2004;81(10):520-523. 
29. Leutscher PD, van Dam GT, Reimert CM, Ramarakoto CE, Deelder AM, Ørnbjerg N. Eosinophil cationic protein, soluble egg antigen, circulating anodic antigen, and egg excretion in male urogenital schistosomiasis. Am J Trop Med Hyg. 2008;79(3):422-426.

30. Kósa L, Kereki E, Farkas M. [Eosinophilic cationic protein levels in feces and serum in children with food allergies]. Orv Hetil. 1997;138(6):345-347. Hungarian.
31. Majamaa $H$, Miettinen A, Laine S, Isolauri E. Intestinal inflammation in children with atopic eczema: faecal eosinophil cationic protein and tumor necrosis factor-alpha as non-invasive indicators of food allergy. Clin Exp Allergy. 1996;26(2):181-187.

\section{Publish your work in this journal}

Research and Reports in Tropical Medicine is an international, peerreviewed, open access journal publishing original research, case reports, editorials, reviews and commentaries on all areas of tropical medicine, including: Diseases and medicine in tropical regions; Entomology; Epidemiology; Health economics issues; Infectious disease; Laboratory science and new technology in tropical medicine; Parasitology; Public health medicine/health care policy in tropical regions; and Microbiology. The manuscript management system is completely online and includes a very quick and fair peer-review system. Visit http://www.dovepress. com/testimonials.php to read real quotes from published authors.

Submit your manuscript here: http://www.dovepress.com/research-and-reports-in-tropical-medicine-journal 